\title{
Performance ANalysis of Spatially CORRELATED CYLINDRICAL ANTENNA ARRAY FOR 3D MASSIVE MIMO
}

\author{
Patrick Danuor ${ }^{1}$ and Emmanuel Ampoma Affum ${ }^{2}$ \\ ${ }^{1}$ Department of Electrical and Electronic Engineering, \\ Ho Technical University, Ho, Ghana \\ ${ }^{2}$ Department of Telecommunications Engineering, Kwame Nkrumah \\ University of Science and Technology, Kumasi, Ghana
}

\begin{abstract}
The spectral efficiency of three-dimensional (3D) massive MIMO is impaired due to the spatial correlation (SC) between closed-spaced elements of antenna arrays (AAs). This paper investigates the impacts of arbitrary $Q$-power variations of the Power Cosine distribution for a precise characterization of the SC. This is done by presenting mathematical expressions characterizing the SC of cylindrical antenna array (CAA) regarding Student's -t, Von Mises and Cosine distributions, for the direction of arrival (DoA) of the electromagnetic waves impinging on the receiving antenna. Unlike the Student's -t and Von Mises distribution, our outcome recorded huge mismatch between theoretical and simulation correlation coefficients for arbitrary Q-power values above ten (10) which also reduced the downlink channel capacity. The practical relevance is that even though higher $Q$-power values are the key parameters for system performance, meticulous selection of arbitrary $Q$-power values above ten (10) is essential to minimize errors regarding channel modelling.
\end{abstract}

\section{KEYWORDS}

Antenna Arrays, Spatial Correlation, Uplink Capacity, Massive MIMO

\section{INTRODUCTION}

With the advent of the Fifth Generation (5G) wireless communication networks, the massive multiple-input multiple-output technology has been proposed as one of the key technologies to drive the 5G communication systems, along with Small Cell Networks (SCN) $[1,2,3,4,5]$. Massive MIMO has attracted a lot of attention in wireless communication as it offers significant data rates through spatial diversity by employing Antenna Arrays (AAs) $[6,7,8,9]$. Antenna arrays (AAs) have become increasingly popular because AAs decide the Spatial Correlation (SC), mutual coupling and channel characteristics, furthering the capacity of massive multiple-input multiple-output (MIMO) systems $[10,11,12,13,14]$. In this paper, we investigate the effect of arbitrary Q-power values of Cosine, Student's - $t$ and Von Misses distribution on the SC of Cylindrical Antenna Array (CAA) for accurate massive MIMO modelling. We derive mathematical expressions for the SC of CAA using the Maximum Power of Arrival (MPA) concept regarding different distributions and study the variations in performance for different values of Q-power due to numerical evaluation of the distribution. The SC of AAs is widely investigated under different angular distributions of arrival. However, different angular distributions present distinct mathematical expressions for the SC [15]. With the progress in massive MIMO innovation, a precise generalization of the SC expressions regarding various 
distributions using direct and exact techniques are vital. The MPA concept offers an efficient method of characterizing the SC and presents exact knowledge of modelling the SC of AAs under different distributions. This prevents the necessity for creating discrete SC expressions under various angular distributions. Until now, investigations of the SC of CAA for higher arbitrary Qpower values of cosine distribution on the channel performance of massive MIMO has received less attention. Authors in [15] derived SC expressions for Q-power cosine distribution. However, no results were presented on the variations of correlation coefficients. With the deployment of massive MIMO, various large-scale antenna architectures have been proposed. The cylindrical antenna array (CAA) array is employed in this paper because the CAA permits the possibility to either generate directed beams in an arbitrary direction in the horizontal plane or to produce an omni-directional pattern and can be employed as a means of clutter suppression via scanning acceleration and space-time signal [16,17]. Also, the radiated massive MIMO signals from these arrays can be well regulated in the three-dimensional (3-D) space to improve the system capacity $[18,19,20]$.

For the objectives of this paper,

1. First, we analyse the 3GPP standard which follows a GBSM approach proposed in $[21,22,23,24]$ and present a new channel realization when the transmitter antenna is represented as CAA.

2. Secondly, we derive expressions for the spatial correlation (SC) characterization of CAA regarding Student's -t, Power Cosine, and Von Mises distributions using the concept of Maximum Power of Arrival (MPA) to investigate the downlink capacity of 3D massive MIMO.

3. Finally, we demonstrate that even though higher Q-power values of the Cosine distribution enhances massive MIMO performance in GBSM, careful selection of Q-power values above ten (10) is vital to minimize errors, as it causes mismatch between theoretical and simulation correlation coefficients. This deteriorates the upper bound downlink channel capacity limits when the transmit antenna increases.

The rest of this paper is organized as follows: Section two (2) presents the proposed 3D of CAA and introduces the concept of Maximum Power of Arrival. Section three (3) presents the system model and the 3D massive MIMO Channel model, which follows a geometry-based stochastic channel model approach. Section four (4) presents the numerical analysis and results of the paper.

\section{Proposed Sc Characterization of caA based on MaXimum POWER OF ARrival}

\subsection{D Sc of CAA using Steering Vectors}

According to [25], the steering vector (SV) for CAA is given as

$$
\mathbf{a}(\theta, \phi)=e^{j R \sin \left(\theta \cos (\delta-\phi)+z_{n} \cos \theta\right)}
$$

where $R$ represents the radius of the circular array and $z_{n}$ is the circular array in the $z$ direction. $\delta$ represents the angular position of the $n^{\text {th }}$ element of the CAA and is given by $\delta=2 \pi(n-1) / N$. The azimuth and elevation angles are bounded between $0 \leq \phi \leq 2 \pi$ and $0 \leq \theta \leq \pi$. 
International Journal of Wireless \& Mobile Networks (IJWMN) Vol. 12, No.5/6, December 2020

According to [25], the angle of arrival, $\varsigma$ is proportional to $\theta \cos (\delta-\phi)+z_{n} \cos \theta$. Following guidelines in [26], the SC of CAA between the real part and that of the imaginary part can be written as

$$
\rho=\left|\int_{\theta} \int_{\phi} \cos \left(e^{j R \sin \left(\theta \cos (\delta-\phi)+z_{n} \cos \theta\right)}\right) P A S(\phi, \theta) d \phi d \theta+j \int_{\theta} \int_{\phi} \sin \left(e^{j R \sin \left(\theta \cos (\delta-\phi)+z_{n} \cos \theta\right)}\right) P A S(\phi, \theta) d \phi d \theta\right|^{2}
$$

where $\operatorname{PAS}(\phi, \theta)$ represents the joint angular distribution of the angle of arrival of both the azimuth and elevation regarding the Power Cosine, Student's - $t$ and Von Mises distributions.

\subsection{Maximum Power of Arrival Concept}

Definition of Maximum Power of Arrival: If $\varsigma$ is the phase angle of the incident wave and $p(u)$ is the true distribution of power as a function of $u=\sin \varsigma$, then the maximum power is relative to the distribution of true power and is expressed as [25]

$$
M P A=\int_{u} p(u) d u
$$

Motivated by the above proposition, the general expression for the SC is obtain by replacing the joint pdf in eqn. (2) by the MPA of the distribution. Thus, the SC expression regarding the MPA following guidelines in [27] is expressed as

$$
\rho=\left|M P A \times \int_{\theta} \int_{\phi} \cos \left(e^{j R \sin \left(\theta \cos (\delta-\phi)+z_{n} \cos \theta\right)}\right) d \phi d \theta+j \int_{\theta} \int_{\phi} \sin \left(e^{j R \sin \left(\theta \cos (\delta-\phi)+z_{n} \cos \theta\right)}\right) d \phi d \theta\right|^{2}
$$

Using guidelines from [16], eqn. (4) can be expressed as

$$
\begin{aligned}
& \rho=\mid M P A \int_{\phi} \frac{a}{a^{2}+1} \cos \left(e^{j R \sin \left(\theta \cos (\delta-\phi)+z_{n} \cos \theta\right)}\right)+\sin \left(e^{j R \sin \left(\theta \cos (\delta-\phi)+z_{n} \cos \theta\right)}\right) d \phi \\
& +\left.\frac{a}{a^{2}+1} j \int_{\phi} \cos \left(e^{j R \sin \left(\theta \cos (\delta-\phi)+z_{n} \cos \theta\right)}\right) d \phi \sin \left(e^{j R \sin \left(\theta \cos (\delta-\phi)+z_{n} \cos \theta\right)}\right) d \phi\right|^{2}
\end{aligned}
$$

This can be expressed in Bessel functions as [26,27]

$$
\rho=\left|\frac{8 a}{a^{2}+1} \times M P A \times \sum_{m=0}^{+\infty} \frac{J_{2 m}(R)}{2 m} \cos \left(2 m \cdot\left(e^{j \sin \theta \sin (\delta+\phi)}\right)_{0, k}\right) \times \sin \left(2 m \cdot \Delta\left(e^{j \sin \theta \sin (\delta+\phi)}\right)_{k}\right)\right|^{2}
$$

where $J_{m}($.$) represents the Bessel function of the first kind and m^{\text {th }}$ order. $N_{c}$ defines the number of clusters.

\subsection{MPA of Power Cosine, Student's -t and Von Mises distributions}

The Power Cosine distribution is expressed [15] as

$$
p(\varsigma)=\left(k_{c} / \pi\right) \cos ^{Q}\left(\varsigma-\varsigma_{m}\right),-\pi / 2+\varsigma_{m} \leq \varsigma \leq \pi / 2+\varsigma_{m}
$$


where $\varsigma$ and $\varsigma_{m}$ are the arrival and the mean arrival angles. Q controls the pdf shape and $k_{c}$ is used to adjust the pdf areas to a unity value. To discover the impacts of AS and ES at all stages of the SC expression, we assume Q is even as in [15]. The MPA regarding the Power Cosine distribution is given in eqn. (7) as

$$
M P A_{\max }(\varsigma)=\left(k_{c} / \pi\right) \int \cos ^{Q}\left(\varsigma-\varsigma_{m}\right) d \varsigma
$$

and is evaluated as

$$
\int \cos ^{2 n} v d \varsigma=\left(1 / 2^{n}\right)\left(\begin{array}{c}
2 n \\
n
\end{array}\right) v+\left(1 / 2^{n-1}\right) \sum_{k=0}^{n-1}\left(\begin{array}{c}
2 n \\
k
\end{array}\right) \times \sin (2 n-2 k) v / 2 n-2 k
$$

According to [15], the Von Mises distribution is expressed as

$$
p(\varsigma)=\left(1 / 2 \pi I_{0}(\kappa)\right) e^{\kappa \cos \left(\varsigma-\zeta_{m}\right)},-\pi+\varsigma_{m} \leq \varsigma \leq \pi+\varsigma_{m}
$$

where $\kappa$ is the accumulation of the distribution, $I_{0}$ is the modified Bessel function. The MPA regarding Von Mises distribution is expressed in eqn. (11) as

$$
M P A_{\max }(\varsigma)=\left(1 / 2 \pi I_{0}(\kappa)\right) \int \mathrm{e}^{\kappa \cos \left(\varsigma-\zeta_{m}\right)} d \varsigma=\left(1 / 2 \pi \kappa I_{0}(\kappa) \sin \left(\varsigma-\varsigma_{m}\right)\right) \mathrm{e}^{\kappa \cos \left(\varsigma-\zeta_{m}\right)}
$$

Likewise, from [15], the Student's -t distribution is given as

$$
P_{s t d}(u)=\frac{1}{2} \frac{\mu^{2}}{\mu^{2}+\left(u^{2}+(u-\mathrm{M})^{2}\right)^{3 / 2}}
$$

where $\mu$ denotes the measure of angular spread and $\zeta$ is the angle of arrival. Applying the MPA concept, the MPA regarding the Student's -t distribution is given in eqn. (13) as

$$
M P A_{\max }^{s t d}(\zeta)=\frac{1}{2 \mu} \int \frac{\cos \zeta}{\left(1+m^{2} \sin ^{2} \zeta\right)^{3 / 2}} d \zeta
$$

\section{SYSTEM MODEL}

\subsection{General Format, Page Layout and Margins}

A downlink 3D massive MIMO system employing several transmit antennas $M$, serving simultaneously $K$ single-antenna users is considered [28,29]. The $K$ terminals receive a $K \times 1$ vector on the downlink where Time Division Duplex (TDD) is assumed as

$$
\mathbf{x}_{f}=\sqrt{\rho_{f}} \bar{H}^{T} \mathbf{s}_{f}+\mathbf{w}_{f}, \bar{H}=R_{t}^{1 / 2} H_{3 D}
$$

where $\mathbf{x}_{f}$ is the received signal at the UE, $\rho_{f}$ denotes the transmit SNR, [.] ${ }^{T}$ represents matrix transpose and $\mathbf{s}_{f}$ defines an $M \times 1$ precoded vector of data symbols. $\mathbf{w}_{f}$ represent a $K \times 1$ noise 
vector with an independent (iid) $\mathrm{CN}(0,1)$ noise vector. $\bar{H}$ represents the correlated channel and $H_{3 D}$ is the three-dimensional (3D) $M \times K$ channel matrix. $R_{t}$ is the $N_{T X} \times N_{T X}$ transmit SC matrix at the transmitter.

\subsection{D Massive MIMO Channel Model}

We examine the 3GPP standard which follows GBSM approach in $[21,22,23,24]$ and present a new channel model when the BS antenna is characterized as CAA. The GBSM standard is supported by existing literature which asserts that the fact that propagation paths in the azimuth only does not enhance performance [30,31]. Again, the 3GPP standard under consideration present the elevation angle of the antenna boresight $\theta_{\text {tilt }}$ into the channel and permits dynamic adaptation of the downtilt angles. This opens up several benefits for 3D beamforming which can lead to substantial system performance [30].

According to [30], the effective 3GPP channel between the BS antenna port, $s^{\text {th }}$ and the MS antenna port $u^{\text {th }}$ is expressed as

$$
H_{3 D}=\sum_{n=1}^{N} \alpha_{n} \sqrt{g_{t}\left(\varphi_{n}, \vartheta_{n}, \theta_{t i l t}\right)} \sqrt{g_{r}\left(\phi_{n}, \theta_{n}\right)} \times\left[a_{t}\left(\varphi_{n}, \vartheta_{n}\right)\right]_{s} \times\left[a_{r}\left(\phi_{n}, \theta_{n}\right)\right]_{u}
$$

where $\alpha_{n}$ is defined as the complex random amplitude of the $n$ path. $u=1, \ldots, N_{M S}$ and $s=1, \ldots, N_{B S}$. $\left(\varphi_{n}, \vartheta_{n}\right)$ represents the azimuth and elevation angles of departure (AoDs), respectively. $\left(\phi_{n}, \theta_{n}\right)$ denote the azimuth and elevation angles of arrival (AoAs), respectively of the $n^{\text {th }}$ path. The gain of the antenna array at the BS is given in $[31,32]$ as

$$
g_{t}\left(\varphi_{n}, \vartheta_{n}, \theta_{t i l t}\right) \approx g_{t, H}\left(\varphi_{n} g_{t}, V\left(\vartheta_{n}, \theta_{t i t t}\right)\right)
$$

To demonstrate our idea, the vertical antenna patterns at the BS is approximated in $\mathrm{dB}$ as in [30],

$$
g_{t, H}(\phi)=-12\left(\frac{\phi}{\phi_{3 d B}}\right)^{2} d B
$$

and the vertical antenna pattern at the BS is approximated in $\mathrm{dB}$ as in [30] as

$$
g_{t, v}\left(\theta, \theta_{\text {tilt }}\right)=-12\left(\frac{\theta-\theta_{\text {tilt }}}{\theta_{3 d B}}\right)^{2} d B
$$

The antenna array response at the transmitter is given by

$$
\left[a_{t}\left(\varphi_{n}, \vartheta_{n}\right)\right]_{s}=\exp \left(i k(s-1) d_{t} \sin \vartheta\right)
$$

and at the receiver, it is represented by

$$
\left[a_{r}\left(\phi_{n}, \theta_{n}\right)\right]_{u}=\exp \left(i k(s-1) d_{r} \sin \phi \sin \theta\right)
$$




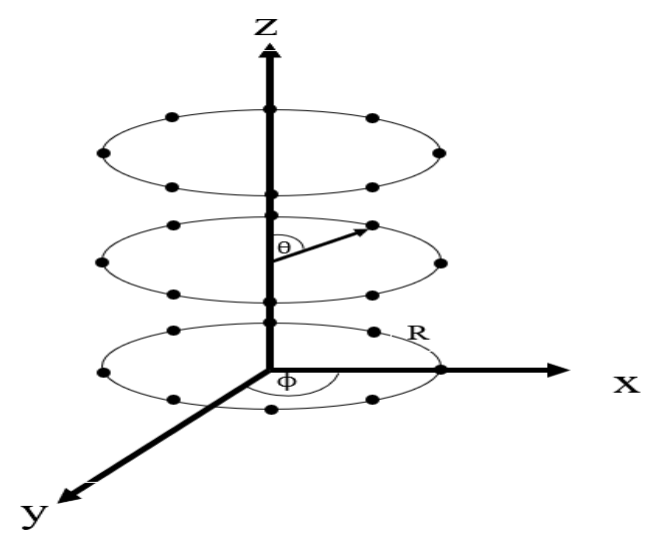

Figure 1. Antenna Configuration of Cylindrical Antenna Array

From Figure 1 above, the angular position of the $n^{\text {th }}$ element of the $m^{\text {th }}$ CAA on the $x, y$ plane is expressed as $\varphi_{s}=2 \pi(n-1) / N$. The location vector can therefore be represented as $v_{t} \cdot x_{s}=\cos \left(\phi-\varphi_{s}\right) \sin \theta$. Hence, the resultant channel realization between the BS antenna port, $s$ and the MS antenna port, $u$ is expressed as

$$
\begin{aligned}
& {\left[H_{3 D}\right]_{C A}=\sum_{n=1}^{N} \alpha_{n} \sqrt{g_{t}\left(\phi_{n}, \theta_{n}, \theta_{t i l t}\right)} \exp (i k \rho(4 \lambda(m-1) / M)} \\
& \left.\times \cos \left(\phi_{n}-\varphi_{s}\right) \sin \theta_{n}\right) \times \sqrt{g_{r}\left(\varphi_{n}, \vartheta_{n}\right)} \exp (i k(u-1) d r \sin \varphi \sin \vartheta)
\end{aligned}
$$

where $k$ represents the wave number and $d r$ is the separation between the RX antenna ports.

\subsection{Proposed Downlink Channel Capacity}

For the downlink upper-bound capacity of the 3D massive MIMO channel, we examine the uncorrelated DL capacity proposed in [33] as

$$
C^{D L} \leq \frac{T_{\text {data }}^{D L}}{T_{\text {coher }}} \log _{2}\left(1+\mathbf{h}^{H}\left(K_{t}^{U E} \mathbf{h}^{H}\right)+K_{r}^{B S} \mathbf{D}_{\mid \mathbf{| h |}}+\frac{\sigma_{B S}^{2}}{p^{U E}} \bar{I}\right) \mathbf{h}
$$

Where $\mathbf{h}$ is the uncorrelated channel, $\mathbf{h}=\left[h_{1}, \ldots, h_{N}\right]^{T}$ and $\quad \mathbf{D}_{|h|^{2}}=\operatorname{diag}\left(\left|h_{1}\right|^{2}, \ldots,\left|h_{N}\right|^{2}\right)$. To investigate the effect of spatial correlation on channel capacity performance, we replace perfect CSI massive MIMO channel, $\mathbf{h}$ in eqn. (22) by the estimated vector, $\bar{H}$ in eqn. (13) and $R_{t}=I$ for the case of the spatially uncorrelated scenario in [33]. $I$ is the identity matrix. The proposed correlated downlink upper-bound capacity is expressed in eqn. (23) as

$$
C^{D L} \leq \frac{T_{\text {data }}^{D L}}{T_{\text {coher }}} \log _{2}\left(1+\bar{H}^{T}\left(K_{t}^{U E} \bar{H}^{T} \bar{H}\right)+K_{t}^{B S} \bar{D}_{|\bar{H}|^{\mid}}+\frac{\sigma_{B S}^{2}}{p^{U E}} \bar{I}\right)^{-1} \bar{H}
$$

where $p^{B S}$ is the DL transmit power, $K_{t}^{B S}$ and $K_{t}^{U E}$ are the impairment parameters at the BS and MS respectively. $\bar{H}$ is the correlated channel in eqn. (14), $D_{|\overline{\mid}|^{2}}=\operatorname{diag}\left(\left|\bar{H}_{1}\right|^{2}, \ldots,\left|\bar{H}_{N}\right|^{2}\right)$ and $\bar{H}=\left[\bar{H}_{1}, \ldots, \bar{H}_{N}\right]^{T}$. 


\section{Numerical Results}

For numerical validation, we substitute the MPA obtained in equations (8), (11) and (13) into the SC expression of CAA in equation (6) to generate the spatial correlation coefficients of the distributions. This is computed over one hundred (100) channel realizations for different values of $Q$. The accumulation of the distribution, $\kappa$ and the measure of the angular spread, $\mu$ for the Von Mises and the Student's -t distribution respectively are each varied for different values. We consider a sixty-four (64) element CAA, and set the mean azimuth-of arrival and mean elevation of arrival at $95^{\circ}$ for all cases. The BS and MS height is considered as $20 \mathrm{~m}$ and $1.7 \mathrm{~m}$, respectively. In spite of the numerical evaluation, results in Figs. 3 and 4 under Student's $-t$ and Von Mises distribution, respectively show a perfect agreement between theoretical SC expression and simulation results for all the variations in the accumulation factor $\kappa$. However, under the Cosine distribution, we observe that higher arbitrary Q-power values above ten (10) presented errors in the numerical results as illustrated in Fig. 2. Moreover, it can be verified that the errors became insignificant at Q-power values under ten (10). In the downlink capacity analysis of the massive MIMO channel, we observe that the Capacity increases as number of transmit antennas, $M$ increases for the Student's -t and Von Mises distribution as shown in Fig. 5a. However, for Qpower values above ten (10), the capacity deteriorates as transmit antennas increases as illustrated in Fig. $5 b$.

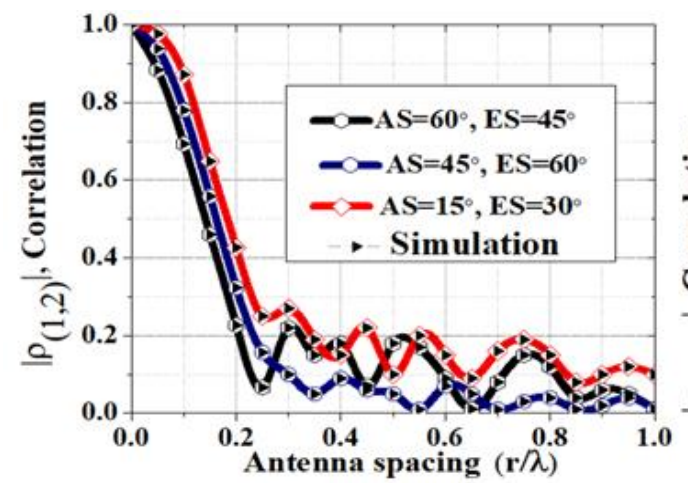

(a)

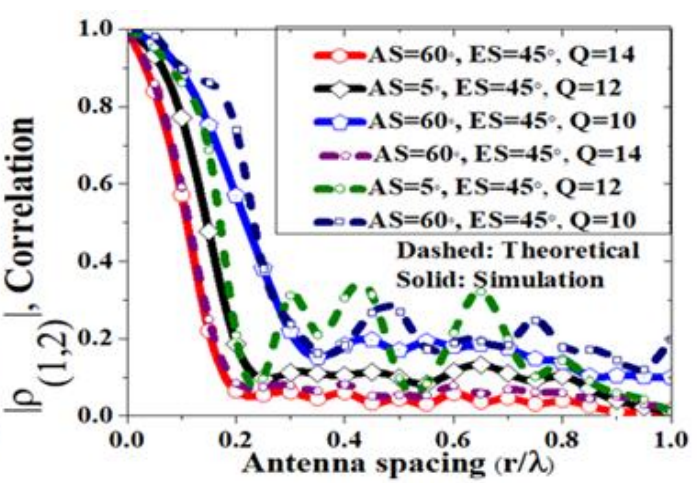

(b)

Figure 2. SC for Q-power Cosine pdf; (a) for $\mathrm{Q}=4$, (b) for $\mathrm{Q}=12$

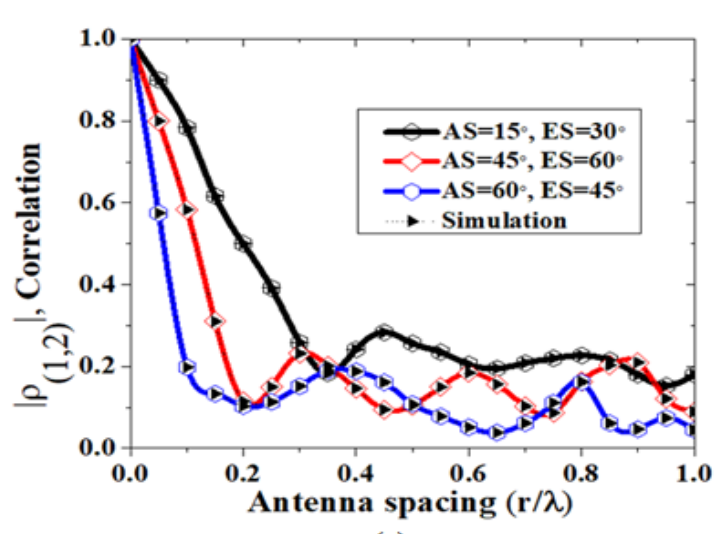

(a)

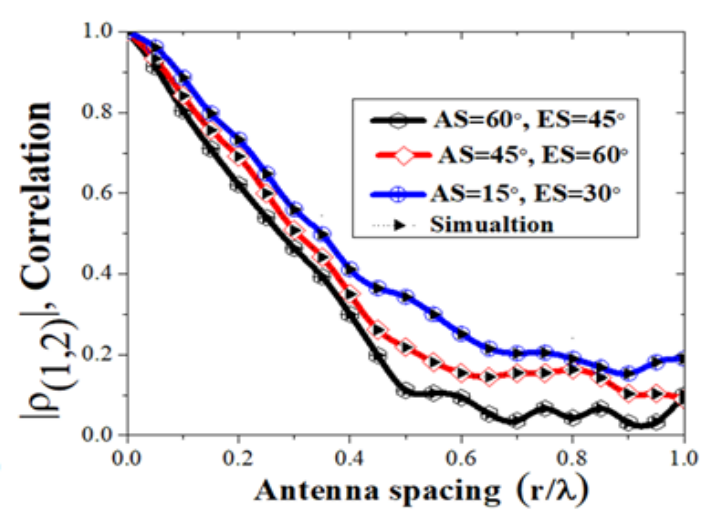

(b)

Figure 3. SC for Von Mises pdf; (a) $\kappa=4$, (b) $\kappa=12$ 


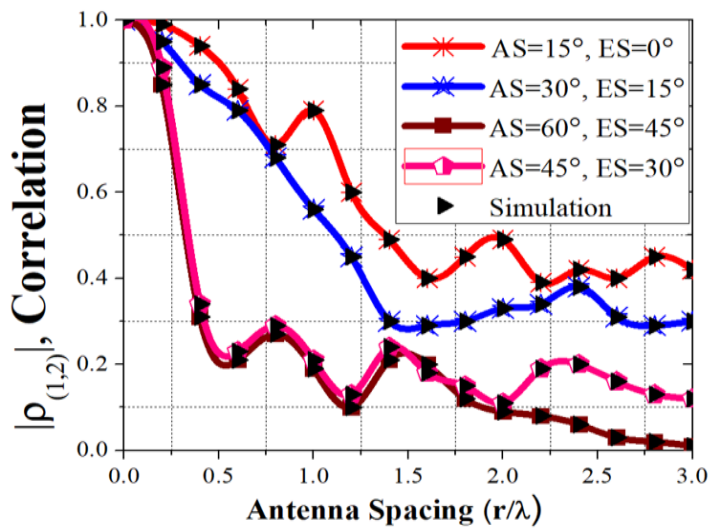

(a)

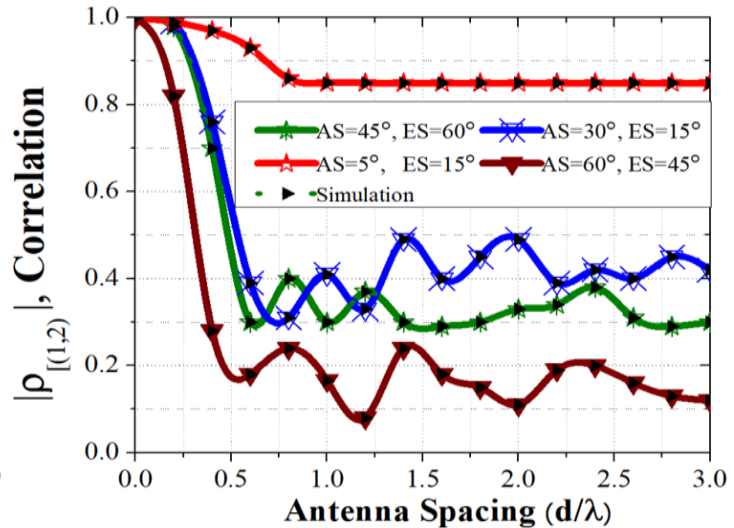

(b)

Figure 4. SC for Student's -t pdf; (a) for $\mu=4$, (b) for $\mu=12$

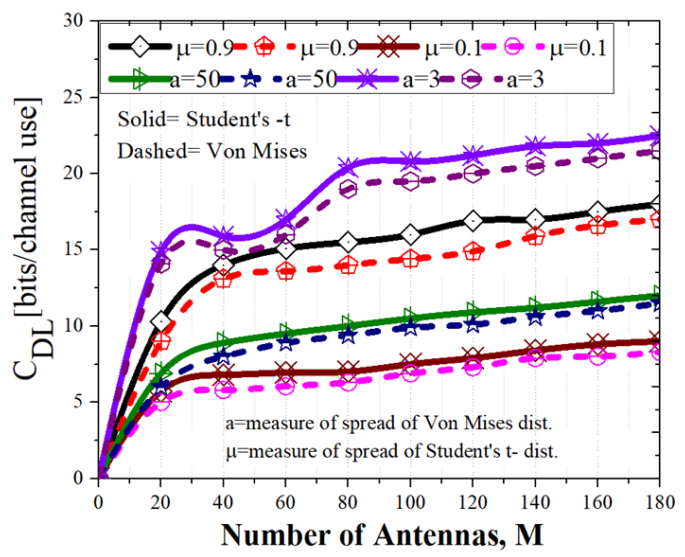

(a)

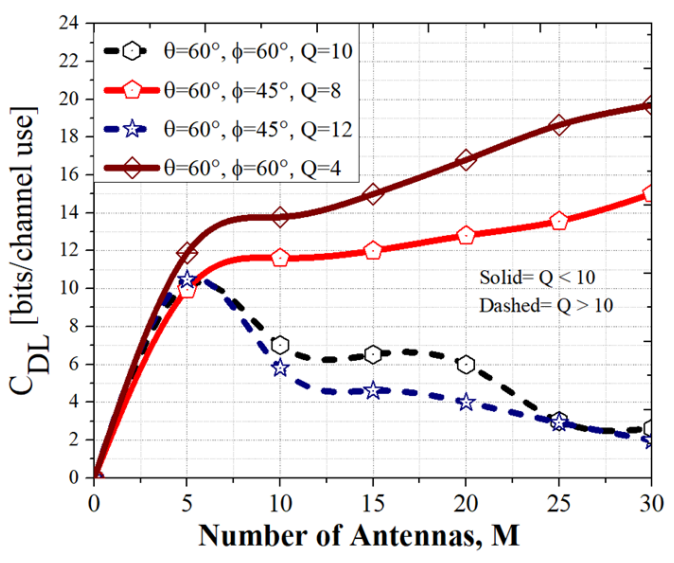

(b)

Figure 5. Comparing the Downlink Capacity for (a) Student's -t and Von Mises dist. and (b) arbitrary Q-Power Cosine dist.

\section{Conclusion}

We have presented the MPA as a precise rule of modelling the SC of antenna arrays regarding different distribution of arrival. We have shown that careful selection of arbitrary Q-power values above ten is necessary for precise characterization of the SC for effective massive MIMO modelling to minimize errors. On the account of the Student's - $t$ and Von Mises distribution, our results support existing fact as perfect agreement was achieved between theoretical and simulation at different values of accumulation factors. Results further indicate that Q-Power values above ten (10) of the Cosine distributions deteriorates the downlink capacity of massive MIMO channels. This work can be further extended to include the effect of the SC of other antenna arrays such as the Spherical Array (SA) regarding various distributions of the angle of arrival.

\section{REFERENCES}

[1] Andrews, J. G., Buzzi, S., Choi, W., Hanly, S. V., Lozano, A., Soong, A. C., \& Zhang, J. C. (2014). What will $5 \mathrm{G}$ be? IEEE Journal on selected areas in communications, 32(6), 1065-1082. 
International Journal of Wireless \& Mobile Networks (IJWMN) Vol. 12, No.5/6, December 2020

[2] Wang, C. X., Haider, F., Gao, X., You, X. H., Yang, Y., Yuan, D., ... \& Hepsaydir, E. (2014). Cellular architecture and key technologies for $5 \mathrm{G}$ wireless communication networks. IEEE communications magazine, 52(2), 122-130.

[3] Osseiran, A., Boccardi, F., Braun, V., Kusume, K., Marsch, P., Maternia, M., ... \& Tullberg, H. (2014). Scenarios for 5G mobile and wireless communications: the vision of the METIS project. IEEE communications magazine, 52(5), 26-35.

[4] Larsson, E. G., Edfors, O., Tufvesson, F., \& Marzetta, T. L. (2013). Massive MIMO for next generation wireless systems. arXiv preprint arXiv:1304.6690.

[5] Agiwal, M., Roy, A., \& Saxena, N. (2016). Next generation 5G wireless networks: A comprehensive survey. IEEE Communications Surveys \& Tutorials, 18(3), 1617-1655.

[6] Rusek, F., Persson, D., Lau, B. K., Larsson, E. G., Marzetta, T. L., Edfors, O., \& Tufvesson, F. (2012). Scaling up MIMO: Opportunities and challenges with very large arrays. arXiv preprint arXiv: 1201.3210 .

[7] Jain, A. K., Acharya, R., Jakhar, S., \& Mishra, T. (2018, April). Fifth Generation (5G) Wireless Technology "Revolution in Telecommunication". In 2018 Second International Conference on Inventive Communication and Computational Technologies (ICICCT) (pp. 1867-1872). IEEE.

[8] Björnson, E., Kountouris, M., \& Debbah, M. (2013, May). Massive MIMO and small cells: Improving energy efficiency by optimal soft-cell coordination. In ICT 2013 (pp. 1-5). IEEE.

[9] Marzetta, T. L. (2010). Noncooperative cellular wireless with unlimited numbers of base station antennas. IEEE transactions on wireless communications, 9(11), 3590-3600.

[10] Manteuffel, D. (2009, November). MIMO antenna design challenges. In 2009 Loughborough Antennas \& Propagation Conference (pp. 50-56). IEEE.

[11] Masouros, C., Sellathurai, M., \& Ratnarajah, T. (2013). Large-scale MIMO transmitters in fixed physical spaces: The effect of transmit correlation and mutual coupling. IEEE Transactions on Communications, 61(7), 2794-2804.

[12] Foschini, G. J., \& Gans, M. J. (1998). On limits of wireless communications in a fading environment when using multiple antennas. Wireless personal communications, 6(3), 311-335.

[13] Yong, S. K., \& Thompson, J. S. (2003, October). A 3-dimensional spatial fading correlation model for electromagnetic vector sensors. In 6th International Symposium on Antennas, Propagation and EM Theory, 2003. Proceedings. 2003 (pp. 843-846). IEEE.

[14] Zhao, L., Yeung, L. K., \& Wu, K. L. (2014). A coupled resonator decoupling network for twoelement compact antenna arrays in mobile terminals. IEEE Transactions on Antennas and Propagation, 62(5), 2767-2776.

[15] Queiroz, W. J., Madeiro, F., Lopes, W. T. A., \& Alencar, M. S. (2011). Spatial correlation for DoA characterization using Von Mises, Cosine, and Gaussian distributions. International Journal of Antennas and Propagation, 2011.

[16] Pluzhnikov, A. D., Pribludova, E. N., \& Ryndyk, A. G. (2014, October). Cylindrical array as a means of the clutter suppression via scanning acceleration and space-time signal processing. In 2014 44th European Microwave Conference (pp. 1864-1867). IEEE.

[17] Sipus, Z., Bosiljevac, M., \& Skokic, S. (2005, July). Mutual coupling analysis of cylindrical waveguide arrays using hybrid SD-UTD method. In 2005 IEEE Antennas and Propagation Society International Symposium (Vol. 1, pp. 155-158). IEEE.

[18] Zheng, K., Ou, S., \& Yin, X. (2014). Massive MIMO channel models: A survey. International Journal of Antennas and Propagation, 2014.

[19] Liu, G., Hou, X., Wang, F., Jin, J., Tong, H., \& Huang, Y. (2016). Achieving 3D-MIMO with massive antennas from theory to practice with evaluation and field trial results. IEEE Systems Journal, 11(1), 62-71.

[20] Balanis, C. A. (2016). Antenna theory: analysis and design. John wiley \& sons.

[21] 3GPP TR 36.873 V12.0.0, "Study on 3D channel model for LTE," Sep.2014.

[22] Zhong, Z., Yin, X., Li, X., \& Li, X. (2013, September). Extension of ITU IMT-advanced channel models for elevation domains and line-of-sight scenarios. In 2013 IEEE 78th Vehicular Technology Conference (VTC Fall) (pp. 1-5). IEEE.

[23] Meinila, J., Kyosti, P., Hentila, L., Jamsa, T., Suikkanen, E., Kunnari, E., \& Narandzic, M. (2010). D5. 3: WINNER+ final channel models. Wireless World Initiative New Radio WINNER. 
International Journal of Wireless \& Mobile Networks (IJWMN) Vol. 12, No.5/6, December 2020

[24] Kammoun, A., Khanfir, H., Altman, Z., Debbah, M., \& Kamoun, M. (2014). Preliminary results on 3D channel modeling: From theory to standardization. IEEE Journal on Selected Areas in Communications, 32(6), 1219-1229.

[25] Andersen, J. B., \& Pedersen, K. I. (2002). Angle-of-arrival statistics for low resolution antennas. IEEE Transactions on Antennas and Propagation, 50(3), 391-395.

[26] Schumacher, L., Pedersen, K. I., \& Mogensen, P. E. (2002, September). From antenna spacings to theoretical capacities-guidelines for simulating MIMO systems. In The 13th IEEE international symposium on personal, indoor and mobile radio communications (Vol. 2, pp. 587-592). IEEE.

[27] H. B. Dwight, Tables of Integrals and Other Mathematical Data. New York, NY, USA: Macmillan, 1957.

[28] Smith, P. J., Neil, C., Shafi, M., \& Dmochowski, P. A. (2014, June). On the convergence of massive MIMO systems. In 2014 IEEE International Conference on Communications (ICC) (pp. 5191-5196). IEEE.

[29] Hochwald, B. M., Marzetta, T. L., \& Tarokh, V. (2004). Multiple-antenna channel hardening and its implications for rate feedback and scheduling. IEEE transactions on Information Theory, 50(9), 18931909.

[30] Kammoun, A., Debbah, M., \& Alouini, M. S. (2015). 3D massive MIMO systems: Modelling and performance analysis. IEEE Transactions on wireless communications, 14(12), 6926-6939.

[31] Kammoun, A., Debbah, M., \& Alouini, M. S. (2016, July). Spatial correlation characterization of a uniform circular array in 3D MIMO systems. In 2016 IEEE 17th International Workshop on Signal Processing Advances in Wireless Communications (SPAWC) (pp. 1-6). IEEE.

[32] Yong, S. K., \& Thompson, J. S. (2003, October). A 3-dimensional spatial fading correlation model for electromagnetic vector sensors. In 6th International Symposium on Antennas, Propagation and EM Theory, 2003. Proceedings. 2003 (pp. 843-846). IEEE.

[33] Björnson, E., Hoydis, J., Kountouris, M., \& Debbah, M. (2014). Massive MIMO systems with nonideal hardware: Energy efficiency, estimation, and capacity limits. IEEE Transactions on Information Theory, 60(11), 7112-7139.

\section{AUTHORS}

Patrick Danuor received the Bachelor of Science (B.Sc.) and Master of Philosophy (MPhil.) degree in Telecommunications Engineering from the Kwame Nkrumah University of Science and technology, Ghana, in 2016 and 2019 respectively. He is an Assistant Lecturer at the Department of Electrical and Electronic Engineering, Ho Technical University, Ghana. His research interests include channel modelling and performance analysis of wireless communication systems.

Emmanuel Ampoma Affum received the B.S. degree in electronics and communication engineering from the Kwame Nkrumah University of Science and Technology, Kumasi, Ghana, in 2007, and the MTech. degree in communication systems from SRM University, Chennai, India. He received his Ph.D. degree with the Centre for RFIC and System Technology, School of Communication and Information Engineering, University of Electronic Science and Technology of China. He is a lecturer at the Department of Telecommunications Engineering, Kwame Nkrumah University of Science and Technology. His current research interests include in the field of wireless communication and signal processing with a particular focus on array processing, channel modelling, and performance analysis of wireless communication systems. 